\title{
Unidimensional measurement may be superior to assess primary tumor response after neoadjuvant chemotherapy for nasopharyngeal carcinoma
}

\author{
Chuanben Chen ${ }^{1,2,3}$, Xiurong Lin ${ }^{1,2,3}$, Yuanji Xu ${ }^{1,2,3}$, Penggang Bai ${ }^{1,2,3}$, Youping Xiao ${ }^{4}$, \\ Yuhui Pan ${ }^{1,2,3}$, Chao Li ${ }^{1,2,3}$, Zhizhong Lin ${ }^{1,2,3}$, Mingwei Zhang ${ }^{5}$ and Yunbin Chen ${ }^{4}$ \\ ${ }^{1}$ Department of Radiation Oncology, Fujian Cancer Hospital, Fujian Medical University Cancer Hospital, Fuzhou, Fujian, China \\ ${ }^{2}$ Shengli Clinical Medical College of Fujian Medical University, Fuzhou, Fujian, China \\ ${ }^{3}$ Fujian Provincial Key Laboratory of Translational Cancer Medicine, Fuzhou, Fujian, China \\ ${ }^{4}$ Department of Radiology, Fujian Cancer Hospital, Fujian Medical University Cancer Hospital, Fuzhou, Fujian, China \\ ${ }^{5}$ Department of Radiotherapy, First Affiliated Hospital of Fujian Medical University, Fuzhou, Fujian, China \\ Correspondence to: Chuanben Chen, email: chenchuanben2010@126.com \\ Keywords: nasopharyngeal carcinoma, neoadjuvant chemotherapy, tumor measurement, magnetic resonance imaging \\ Received: November 22, 2016 Accepted: December 28, 2016 Published: February 01, 2017 \\ Copyright: Chen et al. This is an open-access article distributed under the terms of the Creative Commons Attribution License 3.0 \\ (CC BY 3.0), which permits unrestricted use, distribution, and reproduction in any medium, provided the original author and source \\ are credited.
}

\section{ABSTRACT}

Application of current response evaluation criteria in solid tumors (RECIST 1.1) for assessment of irregularly shaped nasopharyngeal carcinoma (NPC) is a gray area with much ambiguity. Our aim was to compare unidimensional measurements (UDM) and bidimensional measurements (BDM) on magnetic resonance images in alternative planes for measurement of tumor response after neoadjuvant chemotherapy (NACT) in patients with locally advanced NPC. 59 patients with untreated non-metastatic NPC were prospectively enrolled. The size or change in size of the primary tumor and retropharyngeal nodes was assessed by UDM and BDM on axial and coronal planes before and after 2 cycles of NACT. Tumor volume was considered as the reference standard. Correlation between volume and diameter was analyzed using a general linear model. The degree of agreement and discordance of response classification based on different measures were evaluated with $\mathrm{k}$ statistic and McNemar's test, respectively. Both axial UDM (RECIST 1.1) and axial BDM (WHO) showed a significant association with volumetric standard. However, the agreement of axial UDM with VM was better than that of axial BDM ( $K$ value: 0.514 to 0.372 ). In addition, when increasing coronal planes to evaluate tumor response with UDM and BDM, an inferior agreement between coronal BDM and VM was still observed. Notably, coronal UDM showed the best consistency with volume $(\kappa=0.585)$. Hence, axial UDM showed better correlation with volumetric measurements than axial BDM. Since coronal UDM showed high correlation to VM, we suggest further research to assess its use for response assessment of NPC after NACT.

\section{INTRODUCTION}

Nasopharyngeal carcinoma (NPC) represents a particular therioma with irregular infiltration into the surrounding soft tissues, which causes difficulty for clinicians to evaluate therapeutic response. Neoadjuvant chemotherapy (NACT) has repeatedly been shown to reduce the risk of recurrence and distant metastasis in patients with locally advanced NPC [1-3]. Therefore, it is important to precisely evaluate the efficacy of NACT. Currently, assessment of tumor size and change in size in clinical trials is performed based on the World Health Organization (WHO) criteria and the Response Evaluation Criteria in Solid Tumors (RECIST), which is based on bidimensional measurements (BDM) and unidimensional measurements (UDM) on axial planes, respectively $[4,5]$. To our knowledge, the only comparative study of different measures based on response evaluation criteria in NPC 
was conducted by King et al., who found that the BDM to be superior to UDM for evaluation of therapeutic response in patients with irregularly shaped nasopharyngeal tumors [6]. In recent studies, however, UDM was constantly employed to evaluate the efficacy of NACT for NPC, and tumor response to NACT was shown to be closely associated with the prognosis [7, 8]. This has created some confusion over the choice of methods for evaluation of therapeutic efficacy in these patients. Therefore, verification of the practicability of these two techniques is a key imperative.

In 1981, the WHO criteria were first published to estimate tumor size and response by summing the products of bidimensional lesion measurements on axial images [4]. However, some of the parameters and criteria in the WHO standard such as the minimum size of the lesion, the number of lesions recorded, and criteria for disease progression were not well defined. In 2000, the RECIST 1.0 criteria were developed to adopt unidimensional measurement on axial planes to facilitate and refine the tumor response measurement. The RECIST criteria were updated in 2010, with a more detailed definition of measurable and non measurable lesions and disease progression, and to discuss the optimal anatomical assessment of lesions [5]. RECIST guidelines have become more and more popular as its standards are continuously improved to meet the needs of the research. However, their use for evaluation of irregular tumors including malignant pleural mesothelioma and recurrent malignant glioma was not successful [9, 10]. Hence, whether the new RECIST criteria can be applied to assess irregularly shaped tumors remains largely unknown, especially in the era of volumetric measurement.

Although the BDM was shown to be superior to the UDM for assessment of tumor response in patients with NPC [6], some deficiencies existed in this study. First, the number of NPC patients who received NACT was only 17, which limited the statistical power of the analysis. In addition, assessment of retropharyngeal lymph nodes (RLN) was not performed in that study. Some RLNs are difficult to identify as they are often merged with the primary tumor. A close correlation between RLN metastasis and parapharyngeal space involvement as well as metastasis to lower neck nodal levels was reported [11]. Therefore, the approach used by King et al. needs to be modified to include both the measurements of primary tumor and RLN as described in other studies [12, 13]. Furthermore, the techniques are no longer confined to the axial images with increased use of magnetic resonance image (MRI). The alternative plane to use BMD or UDM may also be sagittal or coronal. Therefore, further research is needed to determine which alternative plane may be used to measure primary tumor and RLN in NPC.

In the present study, we use MRI to investigate if the BDM or UDM on the axial plane would authentically reflect tumor size and alteration in size after 2 cycles of NACT in a large cohort of patients with locally advanced
NPC. New measurements such as BDM or UDM on coronal images were assessed with regard to tumor size and change in size with NACT as well. VM served as the reference standard, which was automatically obtained from the $3 \mathrm{D}$ image-based treatment planning system.

\section{RESULTS}

\section{Patient characteristics}

The study group consisted of 59 (39 men and 20 women; median age: 46 y [range, 18-65]) with locally advanced NPC: T1 ( $=9)$; T2 ( $=10)$; T3 $(\mathrm{n}=26)$; T4 (n $=14$ ). Of these, 56 patients had developed retropharyngeal lymph node metastases. The male to female ratio was 1.95:1. Patient characteristics including histopathology, clinical stage, and chemotherapy regimens are summarized in Table 1.

\section{Tumor size at diagnosis}

Tumor volumes at first diagnosis were automatically obtained from the 3D treatment planning system (range: 6.0 to $206.2 \mathrm{~cm}^{3}$; median: $22.7 \mathrm{~cm}^{3}$; interquartile range: $\left.24.1 \mathrm{~cm}^{3}\right)$. The inter-observer reliability for VM, Ax-UDM, Cox-UDM, Ax-BDM, and Cor-BDM is shown in Table 2. The ICCs for VM were significantly higher, and that for Ax-UDM were slightly higher, as compared to Cor-UDM, Ax-BDM, and Cor-BDM, respectively. The correlation of Ax-UDM, Cox-UDM, Ax-BDM, and Cor-BDM with VM is shown in Table 3. All 4 measures showed a significant association with VM at initial diagnosis, according to their probability values.

\section{Evaluation of tumor response}

As for tumor response, for one thing,with regard to absolute change in size and percentage change in size after treatment with NACT, Ax-UDM, Cor-UDM, Ax$\mathrm{BDM}$, and Cor-BDM were still found to be significantly associated with VM (Table 3).

For another, tumor response according to all 4 measures and VM was also evaluated by the $\kappa$ analysis and McNemar's test (Table 4). As for tumor response based on the VM standard, there were $10 \mathrm{PR}$ and $49 \mathrm{SD}$ with no CR and PD. As for tumor response based on Ax-UDM (RECIST 1.1), there were 16 PR and 43 SD, and no $\mathrm{CR}$ and $\mathrm{PD}$, ( $\kappa$ value: 0.514$)$. To compare with the VM standard, Ax-UDM was shown to misclassify 2 PR cases as SD and misclassify $8 \mathrm{SD}$ cases as PR $(P<$ 0.001 , McNemar's test). As for tumor response based on Cor-UDM, there were 14 PR and 45 SD ( $\kappa$ value: 0.585 ). To compare with the VM standard, Cor-UDM was found to misclassify 2 PR cases as SD and misclassify $6 \mathrm{SD}$ cases as PR $(P<0.001$, McNemar's test). As for tumor response based on Ax-BDM (WHO criteria), there were $21 \mathrm{PR}$ and $38 \mathrm{SD}$ ( $\kappa$ value: 0.372 ). When compared with 
Table 1: Characteristics of patients with locally advanced NPC

\begin{tabular}{|c|c|c|}
\hline Parameters & Number of patients & $\%$ \\
\hline \multicolumn{3}{|l|}{ Gender } \\
\hline Male & 39 & 66.1 \\
\hline Female & 20 & 33.9 \\
\hline \multicolumn{3}{|l|}{ Age, years ${ }^{\mathrm{a}}$} \\
\hline$<46$ & 28 & 47.5 \\
\hline$\geq 46$ & 31 & 52.5 \\
\hline \multicolumn{3}{|l|}{ Histopathology ${ }^{b}$} \\
\hline Type II & 4 & 6.8 \\
\hline Type III & 55 & 93.2 \\
\hline \multicolumn{3}{|l|}{$\mathrm{T}$ classification } \\
\hline $\mathrm{T} 1$ & 9 & 15.3 \\
\hline $\mathrm{T} 2$ & 10 & 16.9 \\
\hline $\mathrm{T} 3$ & 26 & 44.1 \\
\hline $\mathrm{T} 4$ & 14 & 23.7 \\
\hline \multicolumn{3}{|l|}{ RLN involvement } \\
\hline Yes & 56 & 94.9 \\
\hline No & 3 & 5.1 \\
\hline \multicolumn{3}{|l|}{ AJCC stage } \\
\hline III & 41 & 69.5 \\
\hline IVa-IVb & 18 & 30.5 \\
\hline \multicolumn{3}{|l|}{ NACT regimen } \\
\hline PTX + nadeplatin & 42 & 71.2 \\
\hline GCB + nadeplatin & 17 & 28.8 \\
\hline
\end{tabular}

Note: ${ }^{a}$ Median 46; range 18-65 years.

${ }^{\mathrm{b}}$ According to the World Health Organization type.

Abbreviations: RLN: retropharyngeal lymph nodes; AJCC: American Joint Committee on Cancer; NACT: neoadjuvant chemotherapy; PTX: paclitaxel; GCB: gemcitabine.

Table 2: Intraclass correlation coefficients for different measurements

\begin{tabular}{lcc}
\hline & ICC (95\%CI) & P-value \\
\hline VM $^{\text {pre }}$ & $0.997(0.996-0.998)$ & $<0.001$ \\
Ax-UDM $^{\text {pre }}$ & $0.969(0.948-0.981)$ & $<0.001$ \\
Cor-UDM $^{\text {pre }}$ & $0.921(0.868-0.953)$ & $<0.001$ \\
Ax-BDM $^{\text {pre }}$ & $0.948(0.913-0.969)$ & $<0.001$ \\
Cor-BDM $^{\text {pre }}$ & $0.945(0.908-0.968)$ & $<0.001$ \\
\hline
\end{tabular}

Abbreviations: ICC: intraclass correlation coefficient; VM: volumetric measurement; Ax-UDM: unidimensional measurements in axial planes; Cor-UDM: unidimensional measurements in coronal planes; Ax-BDM: bidimensional measurement in axial planes; Cor-BDM: bidimensional measurement in coronal planes. 
Table 3: Probability values for associations of different diameter measurements with VM

\begin{tabular}{lccc}
\hline & VM at diagnosis & VM after NACT & $\begin{array}{c}\text { Percentage change in VM } \\
\text { after NACT }\end{array}$ \\
\hline Ax-UDM & $<0.001$ & $<0.001$ & $<0.001$ \\
Cor-UDM & $<0.001$ & $<0.001$ & $<0.001$ \\
Ax-BDM & $<0.001$ & $<0.001$ & $<0.001$ \\
Cor-BDM & $<0.001$ & $<0.001$ & $<0.001$ \\
\hline
\end{tabular}

Abbreviations: VM: volumetric measurement; NACT: neoadjuvant chemotherapy; Ax-UDM: unidimensional measurements in axia planes; Cor-UDM: unidimensional measurements in corona planes; Ax-BDM: bidimensional measurement in axia planes; Cor-BDM: bidimensional measurement in coronal planes.

Table 4: Summary of the kappa values for different measurements

\begin{tabular}{lccc}
\hline & Kappa value & Misclassify SD as PR & Misclassify PR as SD \\
\hline Ax-UDM & 0.514 & 8 & 2 \\
Cor-UDM & 0.585 & 6 & 2 \\
Ax-BDM & 0.372 & 13 & 2 \\
Cor-BDM & 0.381 & 15 & 1 \\
\hline
\end{tabular}

Abbreviations: SD: stable disease; PR: partial response; Ax-UDM: unidimensional measurements in axial planes, CorUDM: unidimensional measurements in coronal planes; Ax-BDM: bidimensional measurement in axial planes; Cor-BDM: bidimensional measurement in coronal planes.

the VM standard, Ax-BDM was found to misclassify 2 $\mathrm{PR}$ cases as SD and misclassify $13 \mathrm{SD}$ cases as PR $(P=$ 0.001 , McNemar's test). As for tumor response based on Cor-BDM, there were 24 PR and 35 SD ( $\kappa$ value: 0.381 ). When compared with the VM standard, Ax-BDM was found to misclassify 1 PR cases as SD and misclassify 15 SD cases as PR $(P<0.001$, McNemar's test $)$. Therefore, it was not difficult to find that the kappa values of the UDM, either on axial or coronal planes, were markedly higher than that of the BDM on axial or coronal planes, and of which, Cor-UDM seemed to be the highest. When VM was taken as the reference standard, Ax-UDM, Cor-UDM, Ax-BDM and Cor-BDM were finally found to misclassify the tumor response in 10 of 59 cases (16\%), 8 of 59 cases (14\%), 15 of 59 cases (25\%), and 16 of 59 cases (27\%), respectively. It was obvious that the BDM measured in alternative planes after NACT accounted for a higher error rate as compared to that with UDM on alternative planes.

\section{DISCUSSION}

Volumetric methods by manual delineation of target areas on 3D cross-sectional images has high repeatability and may provide the most accurate measure of tumor size regardless of the shape of the tumor [14, 15]. Nevertheless, this technique demands a lot of time, a high level of expertise and more manpower that makes it impractical for routine clinical use. New semi-automated measurement technology was used in an effort to make up for these deficiencies, but the results have remained unsatisfactory because of the relatively intensive labor $[16,17]$. Therefore, tumor size was usually assessed by simple diameter measurements including bidimensional measurement (WHO criteria) and unidimensional measurement (RECIST criteria). A previous study indicated that the RECIST criteria (version 1.0) may not be applicable to irregularly shaped nasopharyngeal cancers [6]. However, this trend may be altered with improvements in the new RECIST criteria (version 1.1). Furthermore, taking an alternative plane for UDM or $\mathrm{BDM}$ is likely to become more feasible with widespread use of 3D MR imaging. In the present study, both Axial UDM (RECIST 1.1) and axial BDM (WHO) showed strong correlation with $\mathrm{VM}$ at diagnosis, absolute change, and percentage change on assessment of primary tumor and retropharyngeal nodes in patients with NPC. However, the agreement of tumor response between axial UDM and VM was better than that between axial BDM and VM. In addition, it is noteworthy that the new measurement using coronal UDM was the most consistent with the volumes.

For comparison of tumor size measurements at diagnosis, VM showed the highest ICCs between the observers compared with the other 4 diameter measurements, in spite of their significantly high ICCs. This result revalidated the high reproducibility of VM which could certainly be taken as a reference standard for UMD or BDM of tumor size. In addition, both UDM and BDM showed a significant association with $\mathrm{VM}$ at 
diagnosis, on both axial and coronal MR imaging planes (all $P<0.001$ ). Primary tumor volume was shown to be an independent prognostic factor for local control of NPC, which may appear to be more predictive than T classification [18-20]. However, in recent years, simple diameter measurements were also be used to evaluate the primary tumor volume at diagnosis. Chang et al. found that BDM of primary tumor and retropharyngeal nodes for NPC was also significantly correlated with $\mathrm{VM}$ at first diagnosis on MRI, and that BDM was shown to independently predict distant metastasis and overall survival [13]. Liang et al. reported that UDM for NPC was an independent prognostic factor for all endpoints of disease survival including local control, distant metastasis, and overall survival [21]. Collectively, the simple measurements including UDM or BDM may be further considered for incorporation into the current staging system for NPC to improve the prognostic significance.

With respect to tumor response, all 4 diameter measurements for absolute change in size and percentage change in size after NACT were still significantly associated with VM (all $P<0.001$ ). However, with respect to tumor response assessed by the $\kappa$ analysis and McNemar's test, it was observed that Ax-UDM had a better $\kappa$ value than Ax-BDM (0.514 vs. 0.372), whereas it had lower misclassification rate compared with AxBDM (16\% vs. $25 \%)$. These findings suggest that the current RECIST 1.1 criteria may be superior to WHO criteria for assessment of tumor response to NACT for irregularly shaped NPC. Our results are contrary to those reported by King et al., who found Ax-BDM to be more reliable to reflect tumor response to treatment despite adopting Ax-UDM according to the RECIST 1.0 [6]. All the same, the corresponding explanations have not been well documented in that study. Indeed, the RECIST criteria are based on the theory that the sum of the longest diameters was more coincident with the logarithm of cell number than the sum of bidimensional products [22]. This theory was supported in the context of different types of cancers including both spherical and nonspherical shaped carcinomas such as breast cancer, melanoma, soft tissue sarcoma, and colorectal cancer [22, 23]. Therefore, it may be reasonable to use the RECIST 1.1 criteria to evaluate tumor response to NACT for NPC.

It is interesting to note that Cor-UDM possessed the highest $\kappa$ value of 0.585 , which also illustrated a good agreement with VM. However, Cox-BDM showed inferior concordance to volume with associated $\kappa$ value of only 0.381 . This result was also in line with the previous theory that the longest diameter was a closer indicator of the expression of cell death than the products of bidimensional diameters [22]. To our knowledge, NPC is often characterized by extensive infiltration into the adjacent normal tissues, and the lateral and upward invasion are believed to be the most common invasion patterns for locally advanced tumors [24]. The additional coronal largest diameter was usually aligned with the upward invasion, which might result in a good agreement of tumor response according to VM criteria as well as axial largest diameter along with lateral invasion. Furthermore, coronal images have also been shown to be of additional diagnostic value for primary tumor in rectal cancer [25]. These findings suggest that a combination of axial and coronal images may be better to assess tumor response after NACT for NPC in routine clinical practice, which should be validated in a large-scale research.

A concern in clinical practice is whether the bony tumor invasion should be included in the assessment of tumor response when the skull base is involved by NPC. Due to the mostly persistent abnormalities in the bone marrow on MR images after NACT, King et al. excluded any skull base invasion from the pretreatment and posttreatment measurements in case of underestimation of tumor response [6]. In fact, the latest RECIST 1.1 guidelines have definitely ruled that bone lesions with identifiable soft tissue components on MRI axial planes can be regarded as measurable lesions [5]. Hence, bony tumor invasion was also excluded from the present study unless it met the definition of measurable bone lesions. Another concern is whether RLNs should be included in the assessment of primary tumor because of their frequent embedment in the primary tumor. In the present study, RLNs were ultimately taken into consideration in the assessment of primary tumor size. One reason was that conglomerated RLNs with primary tumor are difficult to be measured with good objectivity and repeatability even if RLNs were commonly considered as the first order nodes that drain the nasopharynx. Furthermore, RLN metastasis was reported to show a good correlation with parapharyngeal space involvement and cervical lymph nodal metastasis [11]. However, whether the regression pattern of RLNs is more linearly related to that of cervical lymph nodes is not known at present. Finally, RLNs were also excluded in the assessment of target lymph nodal response after NACT for NPC in our previous study [26]; it was, therefore, necessary to take RLNs together with primary tumor to evaluate tumor response.

One limitation of the current study is the lack of resected specimens to be accurately measured because radiotherapy serves as the primary therapeutic strategy for NPC rather than surgery. Of course, the measurements of the volumes of the resected specimens were considered as the gold-standard for measurement of tumor size instead of volume measurements on cross-sectional MR images. Nevertheless, volume measurements showed the highest reproducibility when compared with the other four diameter measurements on MRI, which demonstrated that the volume measurements could be taken as a reference standard in the present study. Furthermore, the increasing use of sagittal plane on MRI has proved to be important in the diagnosis and target delineation of NPC [27]; however, the effect of sagittal measurement in the 


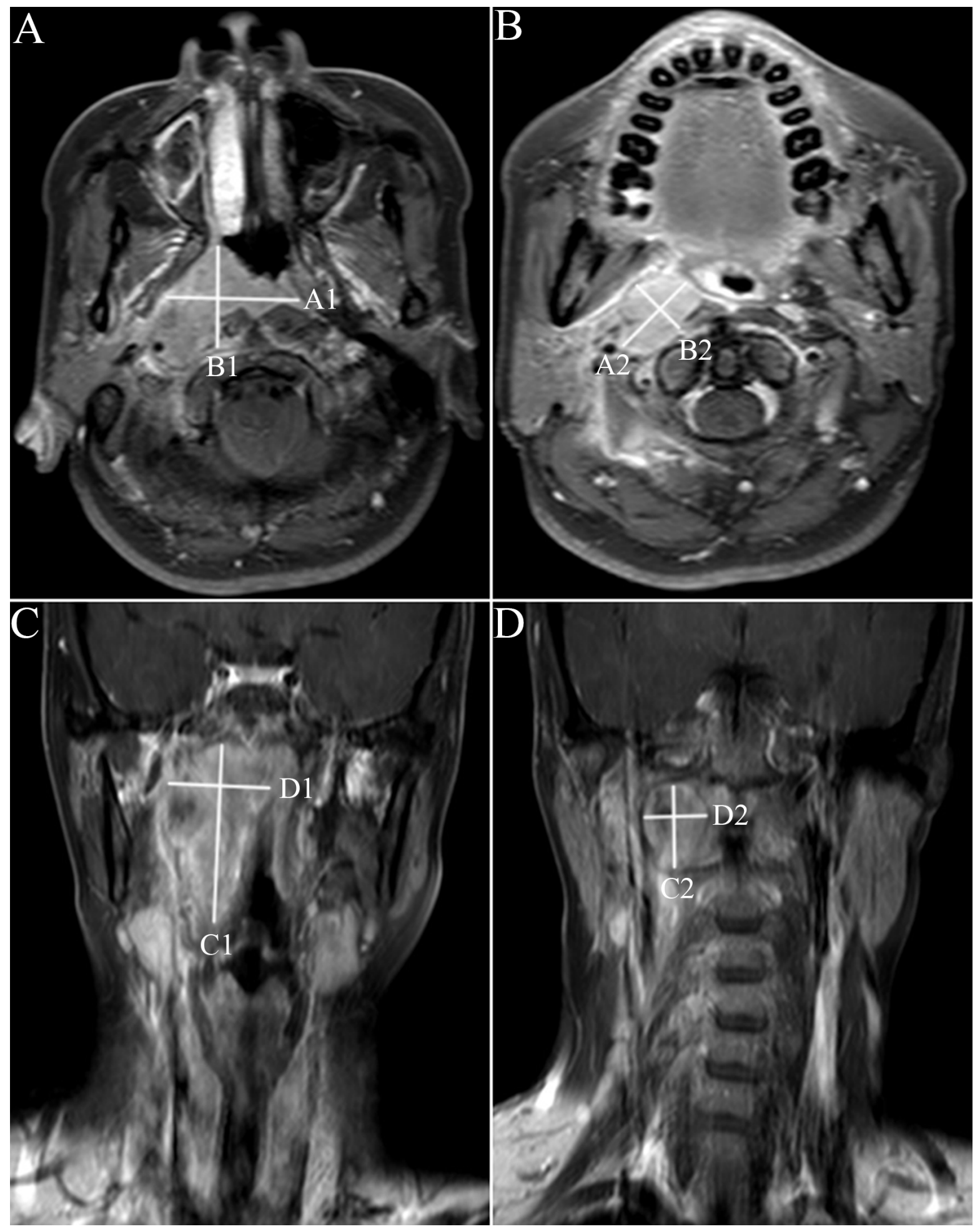

Figure 1: Unidimensional and bidimensional measurements of nasopharyngeal carcinoma in axial and coronal T1weighted postcontrast MR images.

Notes: Ax-UDM was obtained by summation of maximum diameter of the primary tumor A. and retropharyngeal nodes $\mathbf{B}$. in the largest axial slice. Ax-BDM was obtained by summation of the products of the Ax-UDM and the greatest measurement perpendicular to it (A) and retropharyngeal nodes (B). Cor-UDM and Cor-BDM of primary tumor $\mathbf{C}$. and retropharyngeal nodes $\mathbf{D}$. were obtained by the same measurements in the largest coronal slice. Ax-UDM $(\mathrm{cm})=\mathrm{A} 1+\mathrm{A} 2$; Ax-BDM $\left(\mathrm{cm}^{2}\right)=\mathrm{A} 1 \times \mathrm{B} 1+\mathrm{A} 2 \times \mathrm{B} 2 ;$ Cor-UDM $(\mathrm{cm})=\mathrm{C} 1+\mathrm{C} 2$; Cor-BDM $\left(\mathrm{cm}^{2}\right)=\mathrm{C} 1 \times \mathrm{D} 1+\mathrm{C} 2 \times \mathrm{D} 2$

Abbreviations: Ax-UDM: unidimensional measurements in axial planes; Ax-BDM: bidimensional measurements in axial planes; CorUDM: unidimensional measurements in coronal planes; Cor-BDM: bidimensional measurements in coronal planes. 
Table 5: Criteria for the assessment of tumor response

\begin{tabular}{lccc}
\hline Classification & Unidimensional criteria & Bidimensional criteria & Volumetric criteria $^{\mathbf{c}}$ \\
\hline $\mathrm{CR}$ & Tumor disappearance & Tumor disappearance & Tumor disappearance \\
$\mathrm{PR}$ & $>30 \%$ decrease in size & $>50 \%$ decrease in size & $>65 \%$ decrease in size \\
$\mathrm{SD}$ & Size between that for PR and PD & Size between that for PR and PD & Size between that for PR and PD \\
& $>20 \%$ increase in size, the sum & & $>40 \%$ increase in size \\
PD & $\begin{array}{l}\text { increase } \geq 5 \mathrm{~mm} \text {, the appearance } \\
\text { of one or more new lesions }\end{array}$ & $>25 \%$ increase in size & \\
\hline
\end{tabular}

Note: aased on RECIST 1.1 guidelines.

${ }^{\mathrm{b}}$ Based on WHO guidelines.

${ }^{\mathrm{C}}$ According to correlation of alteration in surficial area to alteration in volume.

Abbreviations: CR: complete response; PR: partial response; SD: stable disease; PD: disease progression.

assessment of tumor response has not been investigated. Due to the limitation of the number of slices, the sagittal measurements were excluded from this study. However, it merits further investigation in a future study.

In conclusion, it is appropriate to use Ax-UDM to assess the load or alteration in the size of the primary tumor and retropharyngeal lymph nodes after NACT for NPC. However, Ax-BDM seems not to be applicable in this respect. In addition, Cor-UDM based on MRI is another feasible measurement; however, the importance of Cor-UDM in the evaluation of tumor response warrants further investigation.

\section{MATERIALS AND METHODS}

\section{Patient selection}

This prospective study was approved by the Institutional Ethics Review Boards at the Fujian Provincial Cancer Hospital (Ref. no. 2015-010-02); written informed consent was obtained from all patients. All patients were enrolled at our hospital. The included criteria were: (1) consecutive patients with pathologically proven, and previously untreated non-metastatic NPC stage III$\mathrm{IVb}$ on the basis of the 2010 AJCC Staging System for NPC $[28,29]$; (2) all patients had a Karnofsky score of $\geq 70$, and treatment eligible blood counts (white blood cell count $\geq 3600 / \mathrm{mm}^{3}$, platelet count $\geq 100,000 / \mathrm{mm}^{3}$ ), hepatic function (serum bilirubin $\leq 1.5 \mathrm{mg} / \mathrm{dl}$ ) and renal function (serum creatinine of $\leq 1.6 \mathrm{mg} / \mathrm{dl}$ ); (3) all patients underwent complete MR imaging before and after 2 cycles of neoadjuvant chemotherapy. Patients older than 70 , those younger than 18 , and those with a prior or synchronous malignancy were excluded. A total of 59 patients met the criteria and were enrolled in the study between September 2014 and February 2016.

\section{Chemotherapy}

All 59 patients received 2 or 3 cycles of neoadjuvant chemotherapy according to their disease stage and physical tolerance. Of which, 44 patients with locally advanced NPC received only 2 cycles of NACT, while 15 patients with N3 stage or with T4 disease involved by extensive invasion of skull base or intracranial tissue were given by 3 cycles of NACT. The specific NACT regimens used were as follows: 42 patients were treated with intravenous paclitaxel (135 $\mathrm{mg} / \mathrm{m}^{2}$ on day 1) combined with nedaplatin $\left(80 \mathrm{mg} / \mathrm{m}^{2}\right.$ on day 2) every 3 weeks; 17 patients received intravenous gemcitabine $\left(1000 \mathrm{mg} / \mathrm{m}^{2}\right.$ on day 1 and 8$)$ combined with nedaplatin $\left(80 \mathrm{mg} / \mathrm{m}^{2}\right.$ on day 2$)$ every 3 weeks.

\section{MRI scanning protocol}

The MRI scanning protocol used is detailed elsewhere [26]. Briefly, all patients underwent MRI scans in a 3.0-T MultiTransmit Whole Body scanner (Achieva TX, Philips Healthcare, Best, The Netherlands). All MR images were acquired from the central temporal to the thoracic outlet with a 16-channel head and neck combined coil. The routinely used MRI sequences included axial and sagittal fast-spin echo (FSE) T1-weighted imaging (T1WI), axial and oblique coronal FSE T2WI using SPIR technique, and axial and oblique coronal contrastenhanced (CE) FS T1WI after a bolus injection of 0.1 $\mathrm{mmol} / \mathrm{kg}$ gadolinium with diethylenetriaminepentacetate (Magnevist, Schering AG, Berlin, Germany). The above MR images of each patient were obtained before and after 2 cycles of NACT.

\section{Imaging assessment}

Measurements of primary tumor and retropharyngeal nodes were independently preformed by two radiologists who specialized in NPC with more than 10 years' diagnostic experience of MRI. All measurements were conducted with the picture archiving and communication system (PACS). Retropharyngeal nodes with minimal axial diameter of $\geq 5 \mathrm{~mm}$ at diagnosis were regarded as malignant lesions which required to be measured. UDM was defined as the measurement of the maximum diameter of primary tumor and retropharyngeal nodes in either 
axial or coronal planes (Ax-UDM or Cor-UDM); BDM was defined as the product of the UDM and the greatest measurement perpendicular to the UDM in either axial or coronal planes (Ax-BDM or Cor-BDM) (Figure 1). In the event of skull base involvement without identifiable soft tissue components on pretreatment MRI scans, the bony skull base invasion was regarded as non-measurable lesions, as described in the RECIST 1.1 guidelines. In this case, the portion of bony structures was excluded from our measurement range on both pretreatment and posttreatment scans for the purpose of evaluation of tumor response.

\section{Volumetric measurement}

With regard to the VM, the pretreatment and posttreatment MR images of NACT were first transmitted to the $3 \mathrm{D}$ treatment-planning system. The area of primary tumor and retropharyngeal nodes were also independently delineated by 2 radiation therapists with more than 10 years of experience in the treatment of NPC. In case of the non-measurable targets because of the involvement of skull base, the corresponding portions were not included. Finally, a 3D image of the delineated lesions was automatically generated by the system and volume was automatically calculated as well.

\section{Assessment of tumor response}

The therapeutic efficacy of NACT was assessed by comparing the changes before and after 2 cycles of NACT. According to WHO, RECIST 1.1, and volumetric criteria [4-6], tumor response is usually categorized into 4 types: complete response (CR), partial response (PR), stable disease (SD) and disease progression (PD). The detailed definitions of the above three criteria are summarized in Table 5 .

\section{Statistical analysis}

All date analyses were performed using the SPSS version 17.0 statistical software (SPSS Inc., Chicago, IL, USA). A general linear model with univariate analysis was employed to evaluate potential correlation of VM with Ax-UDM, Cox-UDM, Ax-BDM, and CoxBDM. The correlation coefficient was used to reflect the associations for different diameter measurements with VM. Intra-class correlation coefficient (ICC) was used to assess the test-retest reliability between the different observers for the measurement before and after 2 cycles of NACT. Absolute change and the percentage change were both used to assess tumor response in each group. The tumor response was categorized as CR, PR, SD or PD (Table 5). The degree of agreement of tumor response as assessed by different methods was evaluated by means of $\kappa$ statistic. McNemar's test was used to compare the error rates of different measurements in the assessment of tumor response. A two-tailed $P$ value of $<0.05$ was considered statistically significant for all analyses.

\section{CONFLICTS OF INTEREST}

The authors declare that they have no competing interests.

\section{GRANT SUPPORT}

The statistaical analyses of this study was performed with the help of Fuzhou 211 Project Data Analysis Service Co., Ltd. The present study was funded by the Fujian Provincial Natural Science Foundation (Grant No. 2015Y0010), the Fujian Medical Innovation Program (2014-CX-7), the National Clinical Key Specialty Construction Program, P.R.C, the Key Clinical Specialty Discipline Construction Program of Fujian, P.R.C.

\section{REFERENCES}

1. Sun Y, Li YF, Chen NY, Zhang N, Hu GQ, Xie FY, Sun Y, Chen XZ, Li JG, Zhu XD, Hu CS, Xu XY, Chen $\mathrm{YY}$, et al. Induction chemotherapy plus concurrent chemoradiotherapy versus concurrent chemoradiotherapy alone in locoregionally advanced nasopharyngeal carcinoma: a phase 3, multicentre, randomised controlled trial. Lancet Oncol. 2016; 17:1509-1520.

2. He X, Xu KR, Guo JY, Zhu YL, Liang XM, Liu LX. A meta-analysis of neoadjuvant chemotherapy plus radiatio in the treatment of locall advanced nasopharyngeal carcinoma. J Cancer Res Ther. 2015; 11:C205-C208

3. Wang MM, Tian HM, Li G, Ge TW, Liu YD, Cui JW, Han FJ. Significant benefits of adding neoadjuvant chemotherapy before concurrent chemoradiotherapy for locoregionally advanced nasopharyngeal carcinoma: a meta-analysis of randomized controlled trials. Oncotarget. 2016; 7:4837548390. doi: 10.18632/oncotarget.10237.

4. Miller AB, Hoogstraten B, Staquet M, Winkler A. Reporting results of cancer treatment. Cancer. 1981; 47:207-214.

5. Eisenhauera EA, Therasseb P, Bogaertsc J, Schwartz LH, Sargent D, Ford R, Dancey J, Arbuck S, Gwyther S, Mooney M, Rubinstein L, Shankar L, Dodd L, et al. New response evaluation criteria in solid tumours: revised RECIST guideline (version 1.1). Eur J Cancer. 2009; 45:228-247.

6. King AD, Zee B, Yuen EH, Leung SF, Yeung DK, Ma BB, Wong JK, Kam MK, Ahuja AT, Chan AT. Nasopharyngeal cancers: which method should be used to measure these irregularly shaped tumors on cross-sectional imaging? Int J Radiat Oncol Biol Phys. 2007; 69:148-154.

7. Liu LT, Tang LQ, Chen QY, Zhang L, Guo SS, Guo L, Mo HY, Zhao C, Guo X, Cao KJ, Qian CN, Zeng MS, Bei JX, et al. The prognostic value of plasma Epstein-Barr viral DNA and tumor response to neoadjuvant chemotherapy 
in advanced-stage nasopharyngeal carcinoma. Int J Radiat Oncol Biol Phys. 2015; 93:862-869.

8. Peng H, Chen L, Zhang Y, Li WF, Mao YF, Liu X, Zhang F, Guo R, Liu LZ, Tian L, Lin AH, Sun Y, Ma J. The tumour response to induction chemotherapy has prognostic value for long-term survival outcomes after intensity-modulated radiation therapy in nasopharyngeal carcinoma. Sci Rep. 2016; 6:24835.

9. Dempsey MF, Condon BR, Hadley DM. Measurement of tumor "size" in recurrent malignant glioma: 1D, 2D, or 3D? AJNR Am J Neuroradiol. 2005; 26:770-776.

10. Monetti F, Casanova S, Grasso A, Cafferata MA, Ardizzoni $A$, Neumaier CE. Inadequacy of the new Response Evaluation Criteria in Solid Tumors (RECIST) in patients with malignant pleural mesothelioma: report of four cases. Lung Cancer. 2004; 43:71-74.

11. Tang LL, Li L, Mao YP, Liu LZ, Liang SB, Chen Y, Sun Y, Liao XB, Tian L, Lin AH, Liu MZ, Ma JM. Retropharyngeal lymph node metastasis in nasopharyngeal carcinoma detected by magnetic resonance imaging. Cancer. 2008; 113:347-354.

12. Lee CC, Ho HC, Su YC, Lee MS, Hsiao SH, Hwang JH, Hung SK, Chou P, Lee CC. Bidimensional measurement of nasopharyngeal carcinoma: a simple method to predict outcomes. Clin Otolaryngol. 2009; 34:26-33.

13. Chang TS, Chu ST, Hou YY, Chang KP, Chi CC, Lee CC. Validation of bidimensional measurement in nasopharyngeal carcinoma. Radiat Oncol. 2010; 5:72.

14. Shah GD, Kesari S, Xu R, Batchelor TT, O’Neill AM, Hochberg FH, Levy B, Bradshaw J, Wen PY. Comparison of linear and volumetric criteria in assessing tumor response in adult high-grade gliomas. Neuro Oncol. 2006; 8:38-46.

15. Marten K, Auer F, Schmidt S, Kohl G, Rummeny EJ, Enqelke C. Inadequacy of manual measurements compared to automated CT volumetry in assessment of treatment response of pulmonary metastases using RECIST criteria. Eur Radio. 2006; 16:781-790.

16. Zhou JY, Lim TK, Chong VC, Huang J. Segmentation and visualization of nasopharyngeal carcinoma using MRI. Comput Biol Med. 2003; 33:407-424.

17. Chong VY, Zhou JY, Khoo JB, Huang J, Lim TK. Nasopharyngeal carcinoma tumor volume measurement. Radiology. 2004; 231:914-921.

18. Chong VF, Zhou JY, Khoo JB, Chan KL, Huang J. Correlation between MR imaging-derived nasopharyngeal carcinoma tumor volume and TNM system. Int J Radiat Oncol Biol Phys. 2006; 64:72-76.
19. Chen CB, Fei ZD, Pan JJ, Bai PG, Chen LS. Significance of primary tumor volume and T-stage on prognosis in nasopharyngeal carcinoma treated with intensity-modulated radiation therapy. Jpn J Clin Oncol. 2011; 41:537-542.

20. Liu DB, Long GX, Mei Q, Hu GQ. Primary tumor volume should be included in the TNM staging system of nasopharyngeal carcinoma. Med Hypotheses. 2014; 82:486-487.

21. Liang SB, Deng YM, Zhang N, Lu RL, Zhao H, Chen HY, Li SE, Liu DS, Chen Y. Prognostic significance of maximum primary tumor diameter in nasopharyngeal carcinoma. BMC Cancer. 2013; 13:260.

22. James K, Eisenhauer E, Christian M, Terenziani M, Vena D, Muldal A,Therasse P. Measuring response in solid tumors: unidimensional versus bidimensional measurement. J Natl Cancer Inst. 1999; 91:523-528.

23. Choi JH, Ahn MJ, Rhim HC, Kim JW, Lee GH, Lee YY, Kim IS. Comparison of WHO and RECIST Criteria for response in metastatic colorectal carcinoma. Cancer Res Treat. 2005; 37:290-293.

24. Chen YB, Fang YH, Chen Y, Lu T, Cai LF, Liao J, Chen JY, Hu CM, Zheng DC, Xiao YP, Pan JJ. MRI study Oil the relationship between the invasion patterns and staging of nasopharyngeal. Chinese J Radiol. 2010; 44:1024-1029.

25. Kaur H, Choi H, You YN, Rauch GM, Jensen CT, Hou P, Chang GJ, Skibber JM, Ernst RD. MR imaging for preoperative evaluation of primary rectal cancer: practical considerations. Radioqraphics. 2012; 32:389-409.

26. Chen CB, Zhang MW, Xu YJ, Yue QY, Bai PG, Zhou L, Xiao YP, Zheng DC, Lin KQ, Qiu SF, Chen YB, Pan JJ. Unidimensional measurement may evaluate target lymph nodal response after induction chemotherapy for nasopharyngeal carcinoma. Medicine (Baltimore). 2016; 95:e2667.

27. Rasch CR, Steenbakkers RJ, Fitton I, Duppen JC, Nowak PJ, Pameijer FA, Eisbruch A, Kaanders JH, Paulsen F, Herk MV. Decreased 3D observer variation with matched CT-MRI, for target delineation in nasopharynx cancer. Radiat Oncol. 2010; 5:21.

28. Edge SB, Compton CC. The American joint committee on cancer: the 7th edition of the AJCC cancer staging manual and the future of TNM. Ann Surg Oncol. 2010; 17:1471-1474.

29. Pan JJ, Xu YJ, Qiu SF, Zong JF, Guo QJ, Zhang Y, Lin SJ, Lu JJ. A comparison between the Chinese 2008 and the 7th edition AJCC staging systems for nasopharyngeal carcinoma. Am J Clin Oncol. 2015; 38:189-196. 